\title{
Comparison Between Predicted and Actual Readabilities of EFL Texts
}

\author{
Kusumarasdyati \\ Universitas Negeri Surabaya \\ Surabaya, Indonesia \\ kusumarasdyati@unesa.ac.id
}

\begin{abstract}
The present study attempted to compare the predicted readability computed using formulas and the actual readability expressed by the readers. Forty six students of English Department Unesa ranked five English texts from the hardest to the easiest, then Spearman $r$ was employed to correlate the ranks from the students' perspective with the ranks obtained from the formulas. The results indicated that these two were moderately correlated, suggesting that some texts which were predicted to be hard indeed tend to be considered challenging by the EFL readers.
\end{abstract}

Keywords_reading, readability, EFL

\section{INTRODUCTION}

Effective reading comprehension relies on the interaction between the texts, the writers and the readers. It is a process where the writers communicate ideas to the readers through written texts, and comprehension is considered successful if the readers could understand those ideas intended by the writers. The attempt to understand the ideas is more likely to get easier when the readability of the texts is suitable for the readers. Readability refers to "the level of ease or difficulty with which text material can be understood by a particular reader who is reading that text for a specific purpose" [11]. To estimate the readability of a text, several methods are available. The oldest method is subjective judgment, in which the teacher makes use of his or her intuition to predict whether a text is suitable for the learners' literacy level [4]. Afterwards, in early $20^{\text {th }}$ century word lists were devised to compile high-frequency words, such as Thorndike's The Teacher's Word Book [1]. The texts containing the words included in the lists could be considered easier to comprehend because these words were assumed to be more familiar to the learners. Another method of estimating readability is cloze procedure, which involves deleting every $\mathrm{n}^{\text {th }}$ word from a text and having the learners complete the blanks with exact words [2]. The last-and probably the most popular-method is readability formulas, which are the focus of the present study. Many teachers prefer readability formulas over the other methods due to the convenience in applying them and the objective results, hence the prevalent use.

More than one hundred readability formulas are available (Fry, 2002) but they have one thing in common: using words and sentences as the criteria of text difficulty [9]. One of the readability formulas widely applied in education is Dale-Chall formula, which takes two variables into account to measure readability: word familiarity and sentence length [5,6]. Another formula called Flesch Reading Ease also includes sentence length as a variable, but it uses word length rather than familiarity to estimate the difficulty of a text [8]. According to Flesch Reading Ease, the more syllables there are in a word, the harder the word becomes to understand. Similarly, Fry Graph (Fry, 2002) and SMOG Grading (Mc Laughlin, 1969) make use of word length and sentence length in predicting whether a text is easy to read or not $[9,10]$.

Although the formulas can estimate the readability of English texts, one important question remains: Can they accurately predict the ease of understanding the texts? The present study attempted to provide an answer to this question by comparing the predicted readability computed from the above formulas and the actual readability expressed by the readers. It aimed to find out the relationship between the actual readability from the readers' perspectives and the readability predicted by the following formulas: Flesch Reading Ease Formula, Fry Graph, SMOG Grading and Dale-Chall Formula [14]. The methodology will be explained in the next section. Afterwards, the results of the study and some implications will be presented.

\section{METHOD}

The present study was approached quantitatively, using correlational study as the research design. The population included all third-year students at English Department, Universitas Negeri Surabaya, and they were selected because they had passed at least four reading courses, having sufficient ability to comprehend English texts at intermediate level. The sample was drawn from the population by means of cluster random sampling, in which two classes ( $\mathrm{N}=41)$ were selected randomly from four classes. They read and ranked five texts (Table 1) in terms of difficulty level, ranging from the hardest (1) to the easiest (5).

TABLE I. THE TEXTS

\begin{tabular}{|l|c|c|c|}
\hline \multicolumn{1}{|c|}{ Titles } & Words & Syllables & Sentences \\
\hline (A) Geese & 267 & 360 & 14 \\
\hline (B) Book & 246 & 309 & 10 \\
\hline
\end{tabular}




\begin{tabular}{|l|c|c|c|}
\hline \multicolumn{1}{|c|}{ Titles } & Words & Syllables & Sentences \\
\hline (C) Landslide & 249 & 386 & 10 \\
\hline (D) Sitting & 306 & 395 & 13 \\
\hline (E) Hard Times & 575 & 601 & 25 \\
\hline
\end{tabular}

The difficulty level of the texts were also estimated by using Flesch Reading Ease Formula, Fry Graph, SMOG Grading and Dale-Chall Formula, resulting in four sets of ranks. The ranks assigned by the participants were then correlated with the ranks estimated by the each formula. Spearman Rank was employed to compute the correlation coefficient.

\section{RESULTS AND DISCUSSION}

The results of analysis demonstrated the readability predicted by the formulas turned out to be slightly different from the actual readability from the readers' perspectives (Table II).

TABLE II. READABILITY SCORES AND RANKS

\begin{tabular}{|c|c|c|c|c|c|c|c|c|c|c|}
\hline \multirow{2}{*}{ Text } & \multicolumn{2}{|c|}{ Readers } & \multicolumn{2}{|c|}{ Flesch } & \multicolumn{2}{c|}{ Fry } & \multicolumn{2}{c|}{ SMOG } & \multicolumn{2}{c|}{ Dale-Chall } \\
\cline { 2 - 11 } & $\boldsymbol{X}$ & $\boldsymbol{R}$ & $\boldsymbol{X}$ & $\boldsymbol{R}$ & $\boldsymbol{X}$ & $\boldsymbol{R}$ & $\boldsymbol{X}$ & $\boldsymbol{R}$ & $\boldsymbol{X}$ & $\boldsymbol{R}$ \\
\hline A & 2.05 & 1 & $\begin{array}{c}67 . \\
4\end{array}$ & 3 & 9 & 3 & 8.7 & 2 & 7.4 & 3 \\
\hline B & 2.34 & 2 & $\begin{array}{c}63 . \\
4\end{array}$ & 2 & 9 & 2 & 8.5 & 3 & 7.6 & 2 \\
\hline C & 3.27 & 3 & $\begin{array}{c}43 . \\
6\end{array}$ & 1 & 14 & 1 & 11.7 & 1 & 8.6 & 1 \\
\hline D & 3.46 & 4 & $\begin{array}{c}68 . \\
4\end{array}$ & 4 & 8 & 4 & 7.4 & 4 & 6.8 & 4 \\
\hline E & 3.88 & 5 & $\begin{array}{c}80 . \\
9\end{array}$ & 5 & 7 & 5 & 5.1 & 5 & 5.8 & 5 \\
\hline
\end{tabular}

Note : $\mathrm{X}=$ readability scores $\mathrm{R}=$ Ranks

In general, the readers considered Text A titled "Geese at the Hospital" as the most difficult one to comprehend, whereas the formulas predicted that the hardest one would be Text $\mathrm{C}$ titled "Landslide in East Java." As to Text B ("A Stolen Book"), both the readers and the formulas-except SMOG Grading - agreed that it was the second hardest text to read. Similarly, all formulas and the readers ranked Text D titled "Sitting Can Be Harmful for Your Health" in the fourth place, suggesting that the ease of comprehending this text was quite high compared to the above texts. Finally, both also viewed Text E ("The Benefits of Hard Times") as the easiest of all.

This finding suggested that the formulas could make precise estimation about the texts that the readers would consider easy to comprehend, as shown in the similarities of ranks assigned by the readers and the formulas. Nevertheless, the formulas became less precise in predicting which texts would be harder for the readers to comprehend because the formulas' readability scores seemed to disagree with the readers'. The difference in the accuracy of prediction could be caused by the factors that affect the ease of comprehension on the part of the readers. Sentence length, word length and word familiarity on which the formulas relied on accurately predicted that a par- ticular text would improve the readability. Therefore, a text with fewer unknown words, shorter words and shorter sentences generally facilitated the readers' effort to comprehend it. As the readability became lower and the readers need to put more efforts in grasping meaning from the text, the cause might not be traced back to the linguistic factors such as words and sentences. There might be other factors that played a role in decreasing the readability level of a text, such as lack of schemata, cultural issues, and others.

To find out how accurately each formula predicts the ease of comprehending the texts, the average readability scores obtained from the readers were correlated with the readability scores computed from each formula, yielding coefficients presented in Table III.

TABLE III. CORRELATION BETWEEN READABILITY SCORES FROM THE READERS AND THE FORMULAS

\begin{tabular}{|l|c|c|c|c|}
\hline & Flesch & Fry & SMOG & Dale-Chall \\
\hline Readers & .60 & .78 & .70 & .60 \\
\hline Flesch & - & .88 & .90 & 1 \\
\hline Fry & - & - & .88 & .88 \\
\hline SMOG & - & - & - & .90 \\
\hline
\end{tabular}

There was a positive, moderate correlation between the actual readability expressed by the readers and the readability estimated by the formulas, with coefficients ranging from .78 to .60 . The formula which predicted the readability of texts most accurately was Fry Graph $(r=.78)$, followed by SMOG Index $(\mathrm{r}=.70)$. Flesch Reading Ease and Dale-Chall Formulas $(\mathrm{r}=$ .60) were equally the least accurate of the four formulas, although their ability in predicting the readability was still moderately good.

An attempt to correlate the readability scores from one formula and those from another formula was also made to find out how consistent those formulas in estimating the readability of texts. The most consistent formulas were Flesch Reading Ease and Dale-Chall Formulas as computation indicated a perfect, positive relationship ( $r=1$ ) between them. This was understandable because they utilized very similar criteria in estimating readability, namely, lexical difficulty (word length in Flesch Reading Ease Formula and word familiarity in DaleChall Formula) and sentence length. Other formulas were slightly less consistent with one another but still demonstrated very high, positive relationship. For instance, Fry Graph and Flesch Reading Ease Formula showed very high consistency (r $=.88$ ), and so did Fry Graph and SMOG Grading as well as Fry Graph and Dale-Chall Formula. The consistency between SMOG Grading and the other two-Flesch Reading Ease and Dale-Chall Formulas-was even slightly higher $(r=.90)$. It could be concluded that these four formulas were relatively consistent in measuring the readability of English texts. 
Despite the consistency, it is important to note that these formulas were moderately rather than highly accurate in the measurement. This finding supported the stance that recommends the use of readability formulas in conjunction with other methods because the formulas served only as a rough estimate $[7,12]$. They take only semantic and syntactic complexity into account and disregarded other factors that might contribute to the text difficulty, such as the readers' prior knowledge, the density of ideas, cohesion, organization and format $[11,3]$. Teachers, therefore, should not use the readability formulas as the only tool to find out whether the texts are of the right difficulty level for the learners. Mobilizing their knowledge about literacy and the learners' characteristics, the teachers can rely on their intuition and make subjective judgment about text difficulty. In addition, trying out the texts using cloze procedure to another group of learners with similar characteristics before presenting the texts in the classroom could help the teachers in estimating the readability. Finally, if some up-to-date word lists are currently available, the high-frequency words are helpful for the teachers to predict whether the lexical items in the text are familiar to the learners and contribute to the ease of comprehension.

\section{CONCLUSION}

This study attempted to match the readability of texts predicted by four well-known formulas and the actual readability from the learners' perspective. The finding showed that these two were moderately correlated. Although it was not a perfect match, the formulas predicted the easier texts more accurately than it did the harder texts. Additionally, the formulas had high correlation with each other, probably because they utilized the same criteria in measuring readability, i.e. lexical and syntactic complexity. The implication from these findings relates to how formulas could be applied to in the teaching of reading comprehension. Rather than using formulas as the sole predictor of readability, reading teachers should estimate it by means of several methods for more accurate results.

\section{REFERENCES}

[1] A. Bailin, and A. Grafstein, Readability: Text and Context, Hampshire: Palgrave Macmillan, 2016.

[2] J. R. Bormuth, Cloze Readability Procedure. CSEIP Occasional Report(1), 1967.

[3] B. C. Bruce, A. D. Rubin, and K. S. Starr, Why Readability Formulas Fail. IEEE Transactions on Professional Communication, PC-24, 50-52, 1981.

[4] J. S. Chall, Varying Approaches to Readability Measurement. Revue québécoise de linguistique, 25(1) 23-40, 1996.

[5] E. Dale, and J. S. Chall, A Formula for Predicting Readability. In W. DuBay (Ed.), The Classic Readability Studies (pp. 63-74). Costa Mensa: Impact Information, 2006a.

[6] E. Dale, and J. S. Chall,. A Formula for Predicting Readability: Instructions. In W. DuBay (Ed.), The Classic Readability Studies (pp. 75-94). Costa Mensa: Impact Information, 2006b

[7] W. DuBay, The Principles of Readability. Costa Mesa: Impact Information, 2004.
[8] R. Flesch, A New Readability Yardstick. In W. DuBay (Ed.), The Classic Readability Studies (pp. 99-110). Costa Mensa: Impact Information, 2006.

[9] E. Fry, Readability versus Levelling. The Reading Teacher, 56(3), 286291, 2002.

[10] G. H. Mc Laughlin, "SMOG Grading-a New Readability Formula," Journal of Reading, vol. 12, no. 8, pp. 639-646, 1969.

[11] J. Pikulski, Readability. Boston: Houghton Mifflin, 2003.

[12] M. Ulusoy, "Readability Approaches: Implications for Turkey," International Education Journal, vol. 7, no. 3, pp. 323-332, 2006.

[13] W.H. DuBay, The Principles of Readability, Costa Mesa, California : Impact Information, 2004.

[14] J.G. Graveel and H.A. Fribourg, "Using reading grade level to assess readability of selected plant and soil science textbook," Journal Of Agronomic Education, vol. 16, no. 1, pp. 24-29, 1987.

[15] P. Lennon, Investigating fluency in EFL: A quantitative approach. Language learning, vol. 40, no. 3, pp. 387-417, 1990. 